\title{
Object recognition and pose estimation using appearance manifolds
}

\author{
Zhong-Hua Hao $\cdot$ Shi-Wei Ma
}

Received: 31 December 2012/ Accepted: 28 April 2013/Published online: 28 May 2013

(C) Shanghai University and Springer-Verlag Berlin Heidelberg 2013

\begin{abstract}
Conventionally, image object recognition and pose estimation are two independent components in machine vision. This paper presented a simple but effective method KNN-SNG, which tightly couples these two components within a single algorithm framework. The basic idea of this method came from the bionic pattern recognition and the manifold ways of perception. Firstly, the shortest neighborhood graphs (SNG) are established for each registered object. SNG can be regarded as a covering and triangulation for a hypersurface on which the training data are distributed. Then for recognition task, the determined test image lies on which SNG by employing the parameter " $k$ ", which could be calculated adaptively. Finally, the local linear approximation method was adopted to build a local map between high-dimensional image space and low-dimensional manifold for pose estimation. The projective coordinates on manifold can depict the pose of object. Experiment results manifested the effectiveness of the method.
\end{abstract}

Keywords Object recognition - Pose estimation · Manifold

\section{Introduction}

Object detection and pose estimation are powerful enablers in intelligent vision systems for surveillance, security,

\section{Z.-H. Hao $(\bowtie) \cdot$ S.-W. Ma}

School of Mechatronic Engineering and Automation, Shanghai

Key Laboratory of Power Station Automation Technology,

Shanghai University, Shanghai 200072, People's Republic of China

e-mail: hzhaly1102@yahoo.com.cn entertainment, robotics, autonomous vehicles and human computer interfaces. Statistical learning methods, such as LDA, SVM [1] and ADABOOST [2] are the primary approaches to solve the two problems. However, these methods suffered some drawbacks. The recognition result is not satisfactory since that invariance features are hard to extract when the pose, illumination and other factors vary from scene to scene. Since the negative samples are needed during leaning process, the selection of negative sample data will determine the generalization of the classifier and the classifier model must be trained again when the new kind of object is added to recognize the training data.

Simulating manifold ways of human perception, in this paper, we propose a unified framework and appearance model to address the object recognition and pose estimation problem. Simultaneously, this method could overcome the weak points mentioned above. At the first step, we establish the shortest neighborhood graph (SNG) for each registered training image set, and regard SNG as a covering and triangulation for the hypersurface that training images are distributed on. Secondly, KNN algorithm is applied to SNG for classification. The simplexes characters that divided by SNG are used to determine the parameter " $k$ " in $\mathrm{KNN}$ and calculate the residual error of linear representation by neighborhood images. This step could point out if the test image belongs to neither class that has been trained and the represented weight could also be determined. Then, the local linear approximation method is utilized to build a local map between high-image space and low-dimensional manifold. The projective coordinates on manifold can depict the pose of object in tested image. This method has three characteristics worth being highlighted:

(i) This method integrates recognition and pose estimation work in one framework. 
(ii) This method gives a way to determine parameter " $k$ " adaptively by utilizing the triangulation information. The " $k$ " gives a reasonable area to search in KNN algorithm.

(iii) For pose estimation of test image, this method gives a map from high image space to low pose space by utilizing the neighborhood relationship generated form recognition step.

The rest of this paper is organized as follows. We briefly summarize the most relevant work and introduce our motivation in Sect. 2. In Sect. 3, we present an introduction to SNG-KNN algorithm. In Sect. 4, we describe our algorithm for classification and pose estimation. The experiments are presented in Sect. 5.

\section{Related work and motivation}

\subsection{Related work}

In 2000, Seung and Lee [3] pointed out that the way of human perception was based on manifold. They claimed that the image of object changes gradually in pose, scale, illumination, and other sources of continuous variability would lie on low-dimensional manifolds those were embedded in high image space. To recognize objects, the brain equates all images from the same manifold, and distinguishes images from different manifolds. The trajectory of data motion on the manifold represents the pose change process.

Coupled with this theory, many manifold learning algorithms (MLAs) are proposed, Isomap [4], LLE [5], LE [6], LTSA [7]. Each MLA attempts to preserve a different geometrical property of the underlying manifold. Meanwhile, many researchers have applied manifold methods to solve pose estimation and motion analysis problem. Ptucha and Savakis [8] converted facial feature points of training faces to a low dimensional projection space to form a smooth manifold surface. Afterwards, the facial features of test image are mapped onto the low dimensional projection surface, where regression models robustly estimate pose. Schwarz, et al. [9] utilized several low-dimensional, activity-specific manifolds which are trained to classify the activity in testing human performance video. For recognition area, Vlachos et al. [10] proposed the weighted Isomap method in 2002. This method give different weights to each edge in SNG in order to make the data closer in same class and enlarge in different class. MDS is used to put the data into low dimensional space, and $\mathrm{KNN}$ is used to classify. In 2006, Amuel et al. [11] adopted LLE algorithm to put the training data into low dimension space, and used SVM to distinguish face and non-face image. However, these methods just take MLA as a pre-process method before classification. They still have the same drawbacks as statistical methods.

In 2002, Wang and Chen [12] proposed a new model of pattern recognition called the bionic pattern recognition (BPR), which is based on "matter cognition" instead of "matter classification". This method gave a hopeful way to overcome the drawbacks mentioned above. The mathematical foundation of this method is the principle of homology-continuity (PHC). This principle depicts continuity among samples of the same class, i.e., if two samples are in the same class, the difference between them must be gradually changed. Afterwards, many researchers have improved this approach [13, 14]. However, in these methods, the topological character of training samples must be analyzed by human before using artificial neural networks to cover the training image sets.

\subsection{Motivation}

It is worth noting that the common assumption of BPR and MLA is a low-dimensional manifold embedded in high image space when the object changes gradually. Therefore, if the sampling density is high enough, the dataset with pose change distributes on a hypersurface in high image space. Moreover, because the process of pose variation has some kinds of regularity, e.g., object rotation, human walking, there should be a meaningful low-dimensional manifold hidden in high-dimensional image space.

Based on this assumption, the object recognition task is converted to determine the hypersurface that test image lies on, and the pose estimation is to find out the location of the test image on the low-dimensional manifold which reflects pose variation.

\section{SNG-KNN algorithm}

\subsection{SNG}

In reality, the distribution of data sets is always nonlinear. The Euclidean distance could not depict the intrinsic geometric distribution of the data sets. The purpose of the SNG is to solve this problem. The key point is to estimate the geodesic distance between faraway points, and give only input-space distances. For neighboring points, input-space distance (Euclidean distance) provides a good approximation to geodesic distance. For faraway points, geodesic distance can be approximated by adding up a sequence of Euclidean distance between neighboring points. Because SNG contains all pose transformation of one object, a test image must lie on the corresponding SNG if it belongs to 
this class. Therefore, the SNG could be regarded as a covering of this class (see Fig. 1).

In mathematics, triangulation is a method that divided hypersurface into connected simplexes that are used to analyze the hypersurface's properties. The simplex is $n$-dimensional geometric solid which has $n+1$ vertexes, and each vertexes pair is connected. Images are regard as points in high image space. In SNG, each point connects nearby points by edges. Hence SNG can be seen as a triangulation for the hypersurface that the training data are distributed on (see Fig. 1).

For recognition task, we need to determine the SNG that test image lies on. The KNN algorithm is adopted in this paper. The parameter " $k$ " determining research area plays a critical role to determine the classification result. And how to select " $k$ " properly is remaining a challenging question. Figure 2 shows the parameter " $k$ " increases from $k_{1}$ to $k_{3}$ and the predict result is vastly different. In Fig. 2, two curves Class 1 and Class 2 stand for the hypersurfaces distribution of two class data, respectively, and $k_{1}, k_{2}, k_{3}$ are the different values of parameter " $k$ ". If $k=k_{1}$, test image does not belong to Class 1 and Class 2 neither. If $k=k_{2}$, test image belongs to Class 1 with more probability. If $k=k_{3}$, the test image belongs to Class 1 and Class 2 with same probability. It can be seen that " $k$ " is a critical parameter to predict result.

\subsection{Simplex analysis}

In mathematics, a manifold of dimension $n$ is a topological space such that each point has a neighbourhood which is homeomorphic to an open set of the Euclidean space of dimension $n$. In this paper, the hypersurface is one kind of manifold, and simplex is a local area of manifold, thus the simplex can be analyzed in Euclidean space. Figure 3 utilizes a tetrahedron to stand for $n$-dimensional simplex.

If a test image lies on one SNG, it should be an inner point of one certain simplex. Thus the recognition task is converted to find out a simplex that contains the test image. It can be easily observed from Fig. 3 that the distance from test image to arbitrarily vertex is smaller than the longest edge of simplex. This conclusion does not prove it here. In this paper, we select parameter " $k$ " equal to the longest edge of simplex that has been calculated in SNG.

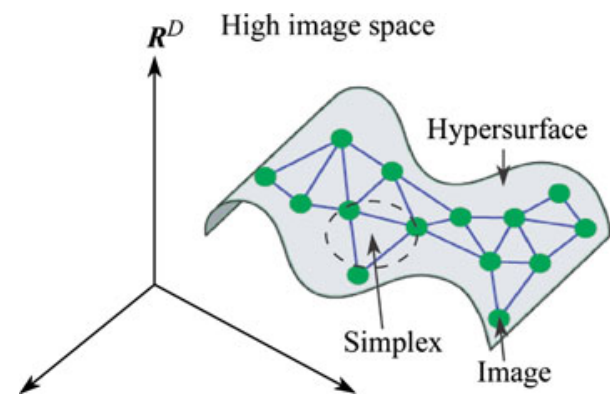

Fig. 1 Covering and triangulation

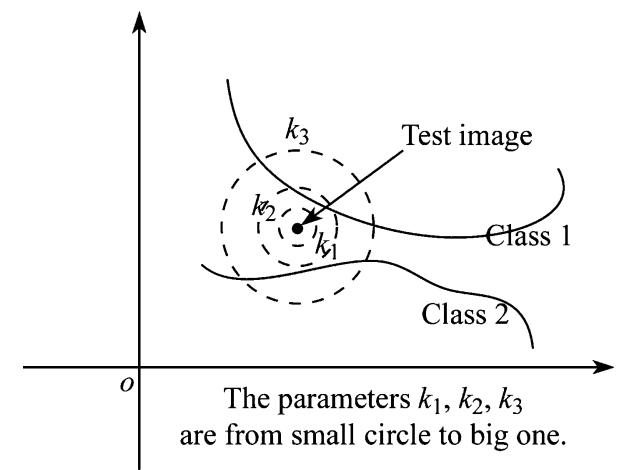

Fig. 2 Parameter " $k$ " selection

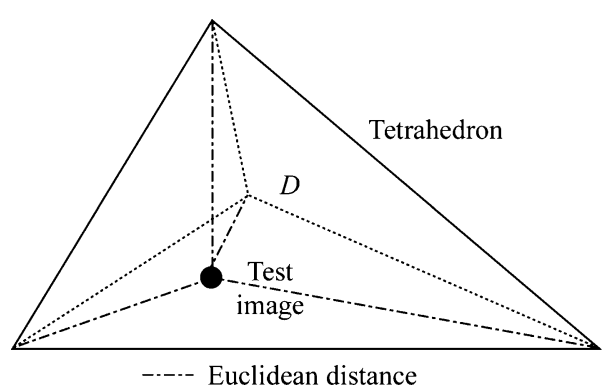

Fig. $3 n$-Dimensional simplex in SNG

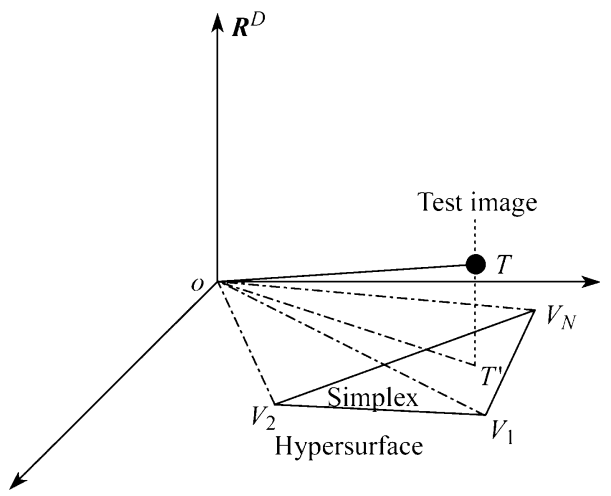

Fig. 4 Location relationship between the test image and simplex

Therefore, if the test image is an inner point, the research area in $\mathrm{KNN}$ must cover the simplex.

However, the research area containing the vertexes of a certain simplex could not guarantee the test image that is an inner point of this simplex. The test image may near the simplex but is not in it. Figure 4 shows this case.

Based on the definition of simplex, the vertexes are linear independent. Therefore, if the test image is an inner point of simplex, it can be represented by linear combination of vertexes. And if the test image is outside of the simplex, the optimum linear representation is projection vector of test image. The more the test image close to simplex, the less difference between the length of test image vector and the length of its projection vector. In 
Fig. 4, the test image and vertexes are defined as $\boldsymbol{T}$ and $\mathbf{v}_{1}, \mathbf{v}_{2}, \cdots, \mathbf{v}_{N}$, respectively. The linear combination that minimize $\varepsilon(W)$ is the projection of test image vector. $e$ is linear combination error which reflects how close the test image to simplex. In the experiment, the test image was considered as an inner point of simplex if $e<0.05$

$$
\begin{aligned}
& \varepsilon(W)=\min \left|\boldsymbol{T}-\sum W_{n} \boldsymbol{v}_{n}\right|^{2}, \quad n=1,2, \cdots, N, \\
& e=\frac{|\boldsymbol{T}|^{2}-\left|\sum W_{n} \boldsymbol{v}_{n}\right|^{2}}{|\boldsymbol{T}|^{2}}, \quad n=1,2, \cdots, N .
\end{aligned}
$$

\section{Method description}

The procedure of the proposed method is shown in Fig. 5. We use the SNG to cover the training images in the same class, and determine the parameter " $k$ " by analyzing simplexes. Then the KNN algorithm was adopted to classify roughly. The $K$ nearest points could also be determined at this step. Using the linear combination of these points to represent the test image and analysis the error to classify precisely, the adopted local linear approximation method can construct a local map between high-dimensional image space and low-dimensional manifold. The projective coordinates on manifold can depict the pose of object in test image.

In high-dimensional image space, a test image corresponds to a point. It can be defined that $T$ is a test image; $C_{n}$ is the data set of class $N ; p_{n-i}$ is the $i$ th data point in

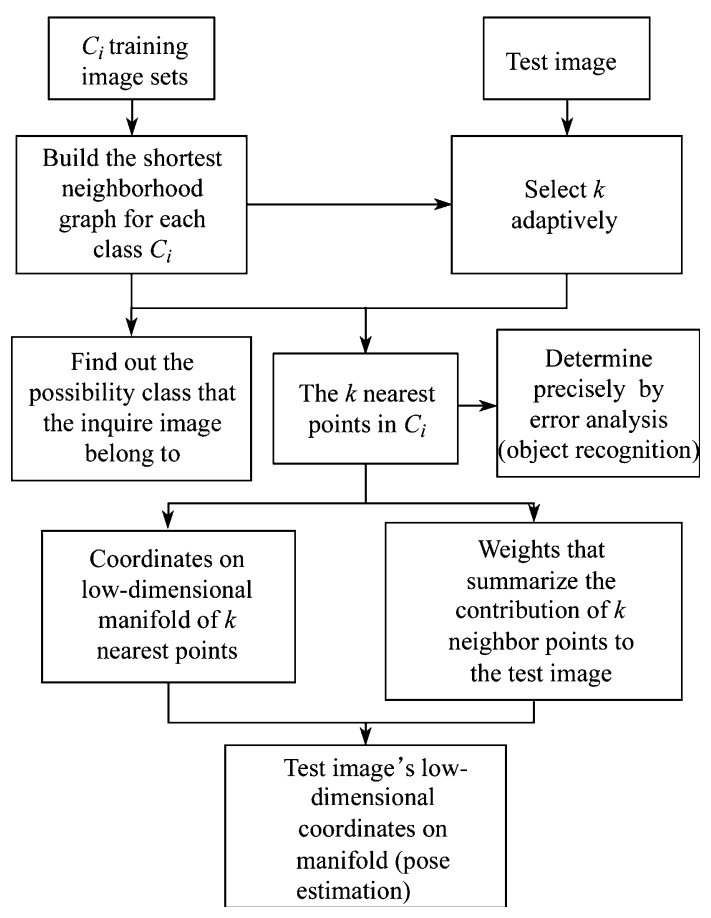

Fig. 5 Flow chart of our new model class $N ; r$ is the radius of a hyperball; $d(i, j)$ is the Euclid distance between point $i$ and point $j$.

\subsection{Object recognition}

There are three steps to object recognition:

(i) Build the $\mathrm{SNG} G_{n}$ for class $C_{n}$,

$$
\begin{aligned}
& d_{n}(i, j)=\left\|\boldsymbol{p}_{n-i}-\boldsymbol{p}_{n-j}\right\|, \\
& G_{n}=\min \left\{d_{n}(i, j), d_{n}(i, h)+d_{n}(h, j)\right\}, \quad h=1,2,3, \cdots, H, \\
& \quad n=1,2,3, \cdots, N,
\end{aligned}
$$

where $\boldsymbol{p}_{n-i}$ and $\boldsymbol{p}_{n-j}$ are the shortest neighbors in $G_{n} ; H$ is the data number in Class $n$; and $N$ is the registered class number.

Select the maximum distance of neighbor points on $G_{n}$ as $k=\max \left\{d\left(\boldsymbol{p}_{n-i}, \boldsymbol{p}_{n-j}\right)\right\}, \quad n=1,2, \cdots, N$.

(ii) Take the test point $T$ as the center of a hyperball and the value of " $k$ " as the diameter. The inner points set covered by the hyperball is

$$
\begin{aligned}
& B(T, r)=\left\{x \in C_{n} \mid d(x, T) \leq r\right\}, \quad r=k / 2, \\
& \quad n=1,2, \cdots, N .
\end{aligned}
$$

If there is no point covered by the hyperball, the test image does not belong to any of classes that have been trained. If $B(T, r)$ is not empty set, the points in it come from $M$ different classes

$T \notin C_{n}, \quad$ if $\{B(T, r)=\phi\}, \quad n=1,2, \cdots, N$.

(iii) Reduce the radius of hyperball. For each class, the radio of the hyperball is selected as

$r_{m}=\max \left\{d\left(\boldsymbol{p}_{m-i}, \boldsymbol{p}_{m-j}\right)\right\}, \quad m=1,2, \cdots, M$.

Find out the points in the hyperball of each class, where $k_{m}$ is the number of point of Class $m$

$$
\left\{\boldsymbol{p}_{m}^{k_{m}}\right\}=\left\{B(T, r) \cap C_{m}\right\}, \quad m=1,2, \cdots, M .
$$

Using the points of each class in hyperball to linearly represent the test image

$$
\begin{aligned}
& \varepsilon_{m}\left(W_{m}\right)=\min \left|T-\sum W_{k_{m}} \boldsymbol{p}_{m}^{k_{m}}\right|^{2}, \quad \sum W_{m}=1, \\
& m=1,2, \cdots, M . \\
& e_{n}=\frac{|T|^{2}-\left|\sum W_{k_{m}} \boldsymbol{p}_{m}^{k_{m}}\right|^{2}}{|T|^{2}}, \quad m=1,2, \cdots, M .
\end{aligned}
$$

The test image belonging to the class $R$ has the minimum $e$, and $e$ must be less than 0.05 


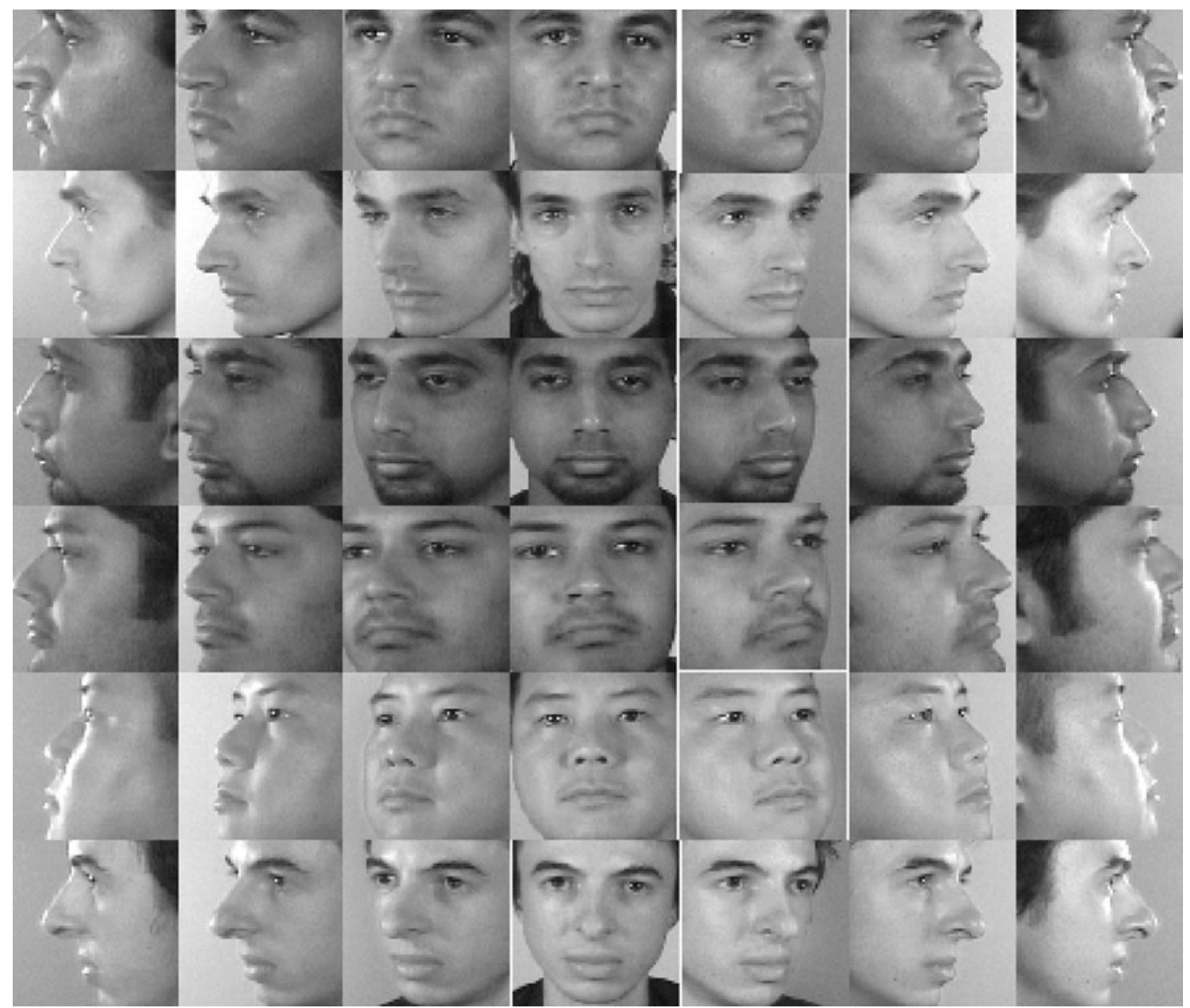

Fig. 6 Examples of facial images

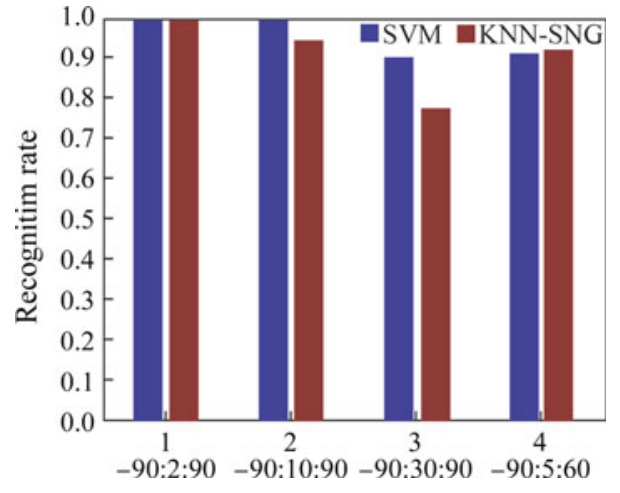

Fig. 7 Performance of SVM and the method in recognition

$T \in C_{\mathrm{R}}, \quad e_{\mathrm{R}}=\min \left\{e_{m}\right\}, \quad m=1,2, \cdots, M$.

In object recognition process, the $k$ nearest points of the test image $k_{\mathrm{R}}$ and the weight $W_{\mathrm{R}}$ summarized the linear contribution error are also determined.

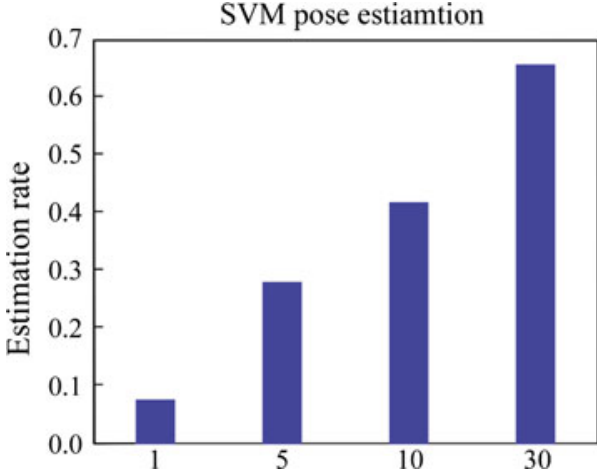

Fig. 8 Performance of SVM in pose estimation under different ranges of face angle changing

\subsection{Pose estimation}

For the pose estimation, we use training image on lowdimensional manifold and find the rule of correspondence between pose and coordinate of an object at the first step. Then the local linear approximation is used to determine 
Table 1 Corresponding relationship between coordinates on low manifold and pose parameter

\begin{tabular}{llllllllllll}
\hline Point & $\boldsymbol{p}_{1-1}$ & $\boldsymbol{p}_{1-2}$ & $\cdots$ & $\boldsymbol{p}_{1-26}$ & $\boldsymbol{p}_{1-27}$ & $t_{2}$ & $\boldsymbol{p}_{1-28}$ & $\cdots$ & $\boldsymbol{p}_{1-50}$ & $t_{1}$ & $\boldsymbol{p}_{1-51}$ \\
\hline Coordinate & 0 & 3.218 & $\ldots$ & 828 & 886.7 & 909.044 & 970.7 & $\ldots$ & 5,999 & $6,410.64$ & 7,385 \\
Degree $\left(^{\circ}\right)$ & -90 & -87 & $\ldots$ & -15 & -12 & -11.202 & -9 & $\ldots$ & 57 & 57.891 & 60 \\
\hline
\end{tabular}

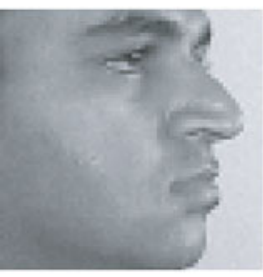

$t_{1}$

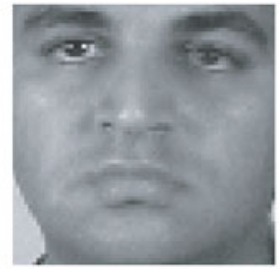

$t_{2}$

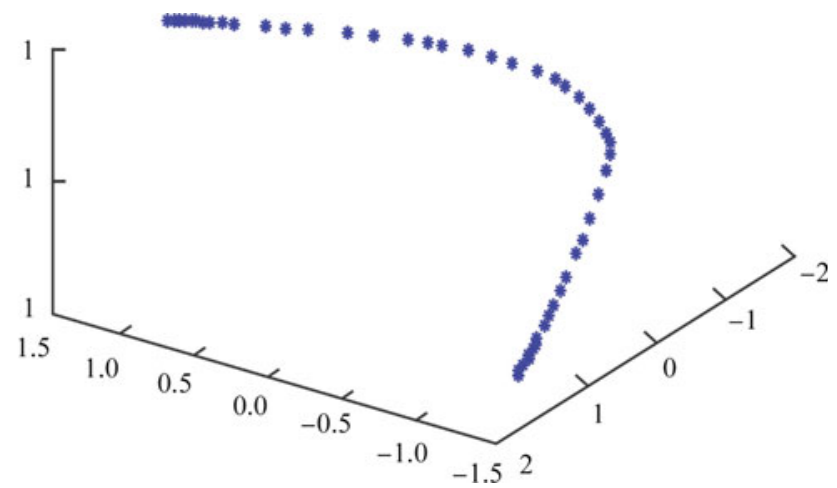

Fig. 9 Test images and dimension reduction result

the coordinate of the test image on manifold. The process is shown as follows.

(i) Using MDS algorithm to project $G_{\mathrm{R}}$ on low-dimensional manifold and get the coordinates of $\boldsymbol{p}_{\mathrm{L}}$.

(ii) Project the test point to a low manifold. This is done by choosing the low-dimensional coordinates of the test point $T_{\mathrm{L}}$ to minimize the embedding cost function.

$\phi\left(T_{\mathrm{L}}\right)=\min \sum\left|T_{L}-\sum W_{\mathrm{R}} \boldsymbol{p}_{\mathrm{L}}\right|^{2}$.

where $T_{\mathrm{L}}$ is $T$ 's coordinate in low-dimensional manifold space, and the pose of the object in test image can be determined by $T_{\mathrm{L}}$.

\section{Experiments and results}

In the experiments, we select FacePix database supposed by Arizona State University. It contains 30 people's face images those are under pose and illumination variation. In our experiment, we select pose variation images of all 30 people for training and test data. For each person, it samples every $1^{\circ}$ while rotating the face from $-90^{\circ}$ to $90^{\circ}$. For all images, we automatically detect the eye position from the first frame. The determined eye position is then used to crop the facial area in the whole image set. The training data is selected from each person at different angels and the rest images are used for testing. Examples of face images are shown in Fig. 6. In Fig. 6, each row contains one person's face image and the column reflect pose change of the head. From left to right column are $-90^{\circ},-60^{\circ},-30^{\circ}$, $0^{\circ}, 30^{\circ}, 60^{\circ}, 90^{\circ}$ respectively.

For comparative study, we use the classical SVM algorithm, which has the best performance among statistical methods.

In recognition process, each person is registered as one class. Therefore, there are 30 classes in our experiment. Each time, we select the different number images as training data in each class to probe the performance of algorithms. The rest images are used for testing. In this paper, "-90:2:90" stands for the training data that the images of one class are selected from $-90^{\circ}$ to $90^{\circ}$ and the neighborhood images are at intervals of $2^{\circ}$. Therefore, there are 91 training images for each class.

Figure 7 shows the performance of our proposed method and SVM under different sample densities of training images. It could be noticed that two methods have extremely high recognition precision with dense sampling. However, the advantage of SVM under sparse sample condition obviously reflected in the second and third subplot. It is also shown that the precise covering established by SNG need high density sampling support. In the last subplot, the performance of our method is a little better than SVM when the training data just cover one part of pose changing process.

In the pose estimation process, we converted the pose estimation problem into classification problem for SVM. Each pose changing area is registered as one class. In our experiment, we select four different ranges of face angle changing, $0-1^{\circ}, 0-5^{\circ}, 0-10^{\circ}$ and $0-30^{\circ}$ respectively. For example, $0-10^{\circ}$ refers to the face rotation divided into 18 classes. The angle changing of the face is within $10^{\circ}$. The 
performance of SVM is shown in Fig. 8. It is obviously that SVM cannot estimate pose precisely. The recognition rate is very low when we want to get precise estimation.

In contrast, our method solves this problem based on manifold learning. It could estimate pose precisely after successful classification.

We illustrate the pose estimation process of two test images of one person in Fig. 8 and Table 1. These two test images are at $58^{\circ}$ and $-11^{\circ}$, respectively in database. Figure 9 shows the result of dimension reduction of the class that test images belong to. The low-dimensional manifold is a curve, because the motion of head rotation just has one degree of freedom. Therefore, the pose of head can be depicted by one-dimensional coordinate. Table 1 list the corresponding relationship between head's angel and low-dimensional coordinate in class one. It is worth to notice that the coordinates are relative in low dimensional space. The results of pose estimation are listed in Table 1. It is obvious that the pose estimation of two images is precise.

\section{Conclusions}

The proposed method deals with the object recognition and poses estimation problem in one integrated framework. Simulating the manifold ways of perception, we use SNG to cover and triangulate of the hypersurface the training image distributed in high image space. Therefore, the recognition task becomes to find the SNG that test image lies on. The object in an image can be correctly recognized when its pose changed largely. Comparing with statistic classification algorithm, this method does not need negative samples and could increase new classes arbitrarily. Different from conventional MLAs such as Isomap and LLE, this method employs local linear approximation algorithm to build a map locally which could project test image on a manifold.

The experiment results on FacePix database show that this new model is effective under condition that object's appearance varies with rotation and any untrained object cannot be recognized incorrectly. Comparing with SVM, this method has better performance on pose estimation. However, it is worth to notice that there are two shortcomings in this method. Firstly, the recognition task is processed in high image space, which has high computation complexity. Secondly, the pose of object just varies in one DOF in database, but the data in real world always has high DOF. These two questions also give a direction for the future work.

\section{References}

1. Moranduzzo T, Melgani F (2012) A SIFT-SVM method for detecting cars in UAV images. In: 2012 IEEE international geoscience and remote sensing symposium (IGARSS), pp 6868-6871

2. Viola P, Jones MJ (2004) Robust real-time face detection. Comput Vision 57(2):137-154

3. Seung HS, Lee DD (2002) The manifold ways of perception. Science 290:2268-2269

4. Tenenbaum JB, de Silva V, Langford JC (2000) A global geometric framework for nonlinear dimensionality reduction. Science 290:2268-2323

5. Roweis LS (2000) Nonlinear dimensionality reduction by locally linear embedding. Science 290:2323-2326

6. Belkin M, Niyogi P (2003) Laplacian Eigenmaps for dimensionality reduction and data representation. Neural Comput 15:1373-1396

7. Zhang Z, Zha H (2004) Principle manifolds and nonlinear dimension reduction via local tangent space alignment. Sci Comput 26(1):313-338

8. Ptucha R, Savakis A (2010) Pose estimation using facial feature points and manifold learning. In: Proceedings of 2010 IEEE 17th international conference on image processing, pp 3261-3264

9. Schwarz LA, Mateus D, Navab N (2012) Recognizing multiple human activities and tracking full-body pose in unconstrained environments. Pattern Recogn 45:11-23

10. Vlachos M, Domeniconi C,Gunopulos D et al (2002) Non-linear dimensionality reduction techniques for classification and visualization. In: Proceedings of 8th ACM SIGKDD international conference on knowledge discovery and data mining, pp 645-651

11. Kadoury S, Levine MD (2006) Finding faces in gray scale images using locally linear embeddings. In: The 5th international conference on image and video retrieval, pp 221-230

12. Wang SJ, Chen X (2003) Biomimetic (topological) pattern recognition: a new model of pattern recognition theory and its application. Neural Netw 3:2258-2262

13. Zeng JY, Zhai YK, Gan JY et al (2009) An effective iris recognition system based on biomimetic pattern recognition. In: International conference on information science and engineering, pp 3583-3586

14. Zhang JB, Cong S, Xu ZM et al (2009) A text classifier based on biomimetic pattern recognition. In: 2009 International conference on machine learning and cybernetics, pp 3463-3468 